\title{
EVALUACION in vitro DE MUTANTES DE CEPAS DE Trichoderma PARA EL CONTROL DE Rhizoctonia solani Y Phytophthora nicotianae EN TOMATE*
}

\section{(In vitro evaluation of Trichoderma strains mutant for the control of Rhizoctonia solani and Phythopthora nicotianae in tomatoes)}

\author{
M. Arias ${ }^{1}$, R. Herrera' ${ }^{1}$, X. Besoaín ${ }^{2}$, \\ L.M. Pérez ${ }^{3}$ \& J. Montealegre ${ }^{1}$ \\ ${ }^{1}$ Dpto. Sanidad Vegetal, U. de Chile, ${ }^{2} \mathrm{P}$. U. Católica de Valparaíso, \\ ${ }^{3}$ U. Andrés Bello. Casilla 1004, Santiago, Chile. mauricio_arias1@yahoo.es
}

Palabras clave: Trichoderma harzianum, T. piluliferum, T. viride, biocontrol, antagonismo, metabolitos Key words: Trichoderma harzianum, T. piluliferum, T. viride, biocontrol, antagonism, metabolites.

\section{RESUMEN}

Se determinó la capacidad antagónica in vitro de mutantes de Trichoderma harzianum (Th), T. piluliferum (Tpi) y T. viride (Tvi), en Rhizoctonia solani cepas 509 (GA 2-1) y 618 (GA 4) y Phytophthora nicotianae cepa 699, aisladas de tomate. Se efectuaron pruebas de antagonismo directo en cultivos duales, metabolitos volátiles y difusibles. Todos los ensayos se desarrollaron a la temperatura y pH óptimos de los 2 patógenos mencionados. Los mutantes se obtuvieron en estudios previos por medio de nitroso guanidinio y luz ultravioleta A $(320 \mathrm{~nm})$ y C $(256 \mathrm{~nm})$, de progenitores previamente caracterizados y seleccionados por su excelente capacidad para expresar mecanismos múltiples de antagonismo en estos patógenos. Todos los mutantes demostraron cierto grado de capacidad antagónica, los mejores correspondieron a los obtenidos con luz ultravioleta A y C, como: Th 11 A 20.1, Th 11 A 80.1, Th 11 A 160.1 y Th 12 A 10.1, provenientes de las cepas de $\mathbf{T}$. harzianum 11 y 12; mientras que para mutantes de nitroso guanidinio el mejor fue Tvi NG 10, de la cepa T. viride. Los resultados obtenidos se evaluarán en ensayos de invernadero y de campo.

\section{INTRODUCCION}

El tomate (Lycopersicon esculentum Mill.) se ha perfilado como uno de los exponentes más importantes de la familia Solanaceae. En Chile presenta una condición

*Parte de la Memoria de Título de Ing. Agr. del primer autor. Financiado a través del Proyecto FONDECYT:1040531-04.
ABSTRACT

The in vitro antagonic capacity of Trichoderma harzianum (Th), T.piluliferum (Tpi) and T.viride (Tvi) mutants was determined in Rhizoctonia solani strains 509 (GA 2-1) and 618 (GA4) and in Phytophthora nicotianae strain 699 isolated from tomatoe. Direct antagonism tests were performed in dual cultures, volatile metabolite and diffusible metabolites. All tests were carried out at the optimum temperature and $\mathrm{pH}$ of the two pathogens mentioned above. Mutants resulted from early studies by means of nitrous guanidinio and UV light A (320 nm) and $C(256 \mathrm{~nm})$, and they came from parents that had been previously characterized and selected for their excellent ability to express multiple antagonism mechanisms in these pathogenous. All mutants showed a certain degree of antagonic capacity, being the best of them those obtained under UV light $A$ and $C$, such as: Th 11 A 20.1, Th 11 A 80.1, Th 11 A 160.1 and Th 12 A 10.1, which came from T.harzianum strains 11 and 12; as to nitrous guanidinio mutants, the best fell in Tvi NG 10 from $\mathbf{T}$. viride strain. Final results will be evaluated in greenhouse and field tests.

de monocultivo, especialmente cuando se realiza bajo invernaderos fríos, cuya superficie alcanza a las 1.073,4 ha, concentrándose su mayor producción entre la IV y VI Regiones (INE, 2001), lo que ha producido un incremento de una serie de enfermedades (CORFO, 1990), que han causando mermas en los rendimientos e inestabilidad de los agroecosistemas debido al inadecuado manejo de los 
problemas relacionados con fitopatógenos e insectos plaga

(Flint \& Roberts, 1988).

Entre los patógenos más importantes que afectan al cultivo en Chile, se encuentran Rhizoctonia solani Künh y Phytophthora nicotianae Breda de Haan, causando enfermedades como el «damping-off» de las plántulas y podredumbre de raíces, cuello y frutos. El control de algunas de estas patologías puede efectuarse mediante la aplicación de tratamientos químicos específicos y fumigación de suelos. Sin embargo, la tendencia se orienta hacia técnicas y procedimientos que garanticen el máximo control de los organismos perjudiciales, sin provocar las alteraciones a nivel ecológico que se generan por el empleo excesivo de agroquímicos (Díaz, 1996).

El uso excesivo de pesticidas químicos y en especial el uso que se le da al bromuro de metilo como herramienta para la desinfección de suelos agrícolas, previo al establecimiento del cultivo, presenta varios inconvenientes, entre ellos la destrucción de organismos benéficos del suelo; no obstante lo anterior, fue su condición de sustancia degradadora del ozono atmosférico, lo que generó la implementación de medidas restrictivas, tanto en su uso, como en su fabricación (Ristaino \& Thomas, 1997). Por esta razón en el año 1997, al firmarse el Protocolo de Montreal, se estableció que el 2005 sería el plazo final para el uso del bromuro de metilo en los países desarrollados. Los países en desarrollo pueden seguir usándolo hasta el 2015, pero disminuyendo en $20 \%$ su línea base (máxima importación permitida). Por lo tanto, Chile deberá finalmente ajustar sus importaciones, reduciéndolas anualmente en un $20 \%$. En concreto, en el 2005 sólo se pudo importar 283 toneladas, casi un 50\% menos que la necesidad del país (Errázuriz, 2005).

Estas razones llevan a buscar alternativas ecológicas que no perjudiquen al hombre ni al medio ambiente, que sean económicamente rentables y capaces de controlar eficazmente a los patógenos. Una de estas alternativas es el control biológico, para que este llegue a ser un componente importante en el manejo de enfermedades vegetales, debe ser efectivo, confiable, consistente y económico. Para alcanzar estos criterios, una de las alternativas es desarrollar cepas de microorganismos más eficientes junto con sistemas de aplicación que aumenten la actividad biocontroladora. Los atributos existentes de control biológico, pueden ser incrementados mejorando los agentes de biocontrol conocidos, mediante su manipulación genética. Esta, puede no sólo incrementar la actividad de los biocontroladores, sino, extender su espectro de acción. Es importante mencionar que los aislados silvestres de $\boldsymbol{T}$. harzianum ya seleccionados, logran de por sí un buen efecto de control, aunque menor al bromuro de metilo (Montealegre et al. , 2002; Pérez et al., 2002), el que podría ser mejorado sustancialmente con cepas mutantes.

Considerando los antecedentes expuestos el objetivo de esta investigación fue: determinar la capacidad antagónica in vitro de mutantes de Trichoderma spp. aislados de tomate, que potencialmente se puedan utilizar como biocontroladores de $\boldsymbol{R}$. solani y $\boldsymbol{P}$. nicotianae.

\section{MATERIALES Y METODOS}

Se utilizaron cepas aisladas de tomate de $\boldsymbol{R}$. solani 509 (GA2-1) y 618 (GA4) y P. nicotianae 699, del cepario del Laboratorio de Microbiología del Departa-mento de Sanidad Vegetal, de la Facultad de Ciencias Agronómicas de la Universidad de Chile.

Los antagonistas mutantes empleados correspondieron a las cepas de $\boldsymbol{T}$. harzianum 11 y 12, obtenidos en la Pontificia Universidad Católica de Valparaíso mediante el uso de luz ultravioleta A (320 nm) y C (256 nm) (Besoaín et al., 2004), y mutantes de T. harzianum 650 y T. viride, a partir de nitroso guanidinio, obtenidos en la Universidad Andrés Bello (Pérez et al., 2004), cuyos progenitores fueron previamente caracterizados y seleccionados por su excelente capacidad para expresar mecanismos múltiples de antagonismo contra los patógenos $\boldsymbol{R}$. solani y $\boldsymbol{P}$. nicotianae (Montealegre et al. , 2002; Besoaín et al., 2001; Pérez et al., 2002).

Los antagonistas y las cepas de $\boldsymbol{R}$. solani y $\boldsymbol{P}$. nicotianae, se hicieron crecer a sus $\mathrm{pH}$ y temperaturas óptimas (García, 2000; Madrid, 2002) (Tabla 1). Los diferentes $\mathrm{pH}$ se lograron agregando $\mathrm{NaOH}$ o $\mathrm{HCl}$ diluidos al medio de cultivo Agar Papa Dextrosa (APD) según se requirió, midiendo permanentemente con un peachímetro (Hanna Instruments HI 9321 Microprocessor) antes de autoclavar el medio de cultivo. La temperatura óptima se obtuvo cultivando las cepas de los patógenos en cámaras de cultivo individuales, ajustadas a la requerida para cada cepa.

\section{Antagonismo directo a través de cultivos duales:}

Discos de APD de $5 \mathrm{~mm}$ de diámetro con el antagonista mutante y con el patógeno, obtenidos con un sacabocados desde los márgenes de cultivos de 4 días de incubación, se colocaron frente a frente a $4 \mathrm{~cm}$ y en forma equidistante del centro de la placa, luego las placas se incubaron a la temperatura y $\mathrm{pH}$ óptimos de cada patógeno (Tabla 1), realizándose cuatro repeticiones para cada combinación patógeno/mutante. Los tratamientos testigos consistieron sólo en un disco de micelio del patógeno creciendo sobre el medio APD. La evaluación se efectuó cuando el crecimiento del testigo cubrió toda la placa, momento en que se determinó el porcentaje de inhibición de crecimiento radial (ICR) mediante la fórmula de Dennis 
Tabla 1. Temperaturas y pH óptimos de los patógenos utilizados (García, 2001* y Madrid, 2002**)

\begin{tabular}{|c|c|c|}
\hline Especies & pH & $\mathbf{T}^{\circ}$ \\
\hline R. solani 509 GA 2-1** & 7,0 & $20^{\circ} \mathrm{C}$ \\
\hline R. solani $618 \mathrm{GA} 4^{* *}$ & 6,5 & $22^{\circ} \mathrm{C}$ \\
\hline P. nicotianae ${ }^{*}$ & 6,0 & $30^{\circ} \mathrm{C}$ \\
\hline
\end{tabular}

y Webster (1971a): Donde R1 es la distancia más lejana recorrida por el patógeno y R2 es la distancia recorrida por el patógeno hacia el antagonista.

\section{ICR (\%) = (R1-R2) 100/R1}

\section{Formación de metabolitos volátiles:}

Se evaluó la producción de metabolitos volátiles en base al método de Dennis y Webster (1971b). En placas de Petri con medio APD se colocaron centralmente discos de $5 \mathrm{~mm}$ de diámetro del patógeno, por otro lado, en placas con el mismo medio, se sembraron discos de igual diámetro (5 mm) con el mutante. Luego se unió cada placa del patógeno con una placa invertida del mutante, y se sellaron con papel parafilm en los bordes. De este modo ambos hongos se dejaron crecer a la temperatura y $\mathrm{pH}$ óptimos del patógeno (Tabla 1) y eventualmente interactuaron sus metabolitos volátiles. Los tratamientos testigos consistieron en un disco de micelio del patógeno creciendo sobre el medio APD unido a una placa sólo con el medio de cultivo. La evaluación consistió en observar y medir el crecimiento radial del patógeno al momento que el testigo cubrió toda la cápsula de Petri.

\section{Formación de metabolitos difusibles:}

Este ensayo se diseñó sobre la base del método de Dennis y Webster (1971b). Las placas de Petri con medio de cultivo se tapizaron con papel celofán estéril (sin film impermeabilizante), en forma de círculos de diámetro inferior al de la placa. Luego se depositó en el centro de ella un disco de cultivo de $5 \mathrm{~mm}$ de diámetro del hongo antagonista. Cuando el antagonista creció lo suficiente, antes de llegar al borde del papel celofán, se retiro con una pinza el papel celofán más el antagonista incluido, cuidando que no hubiese ningún contacto entre el hongo y la superficie del agar y que el antagonista no se encontrara esporulado y en su lugar se colocó un disco de $5 \mathrm{~mm}$ de diámetro de un cultivo de los patógenos. Los tratamientos testigos consistieron en un disco de micelio del patógeno, creciendo sobre el medio APD que contenía papel celofán. La evaluación consistió en observar y medir con un piedemetro, el crecimiento radial del patógeno una vez que el testigo cubrió toda la superficie de la cápsula de Petri.

\section{Diseño estadístico:}

Se utilizó un diseño completamente al azar en que los tratamientos correspondieron a los diferentes antagonistas con 4 repeticiones por cada tratamiento, se utilizó como testigo al patógeno sin interacción. Todos los resultados obtenidos en porcentaje fueron modificados mediante transformación angular Bliss, los cuales se analizaron mediante análisis de varianza (ANDEVA) y en el caso donde existieron diferencias estadísticas significativas se realizó la prueba de Rango Múltiple de Tuckey.

\section{RESULTADOS Y DISCUSION}

Del total de mutantes evaluados, se seleccionaron los mejores para realizar las pruebas de antagonismo directo, metabolitos difusibles o metabolitos volátiles.

\section{Antagonismo directo a través de cultivos duales:}

$\mathrm{Al}$ evaluar los resultados del experimento de antagonismo directo entre los mutantes obtenidos a partir de luz ultravioleta A y C, destacan los mutantes Th 11 A 80.1 y Th 11 A 160.1 en el control de R.solani 509 (GA 21) y 618 (GA 4) ya que estos superan estadísticamente a la cepa silvestre en el control de este patógeno. Para $\boldsymbol{P}$. nicotianae los mutantes solo igualaron el efecto de sus cepas parentales (Tabla 2).

En esta prueba también se pudo apreciar que, los mutantes no tienen un buen efecto directo para la cepa $\boldsymbol{P}$. nicotianae (Tabla 2), esto también fue observado por KûcûK y Kivanc (2003), cuando el aislado de $\boldsymbol{T}$. harzianum cepa T19 inhibió de mejor forma a los patógenos $\boldsymbol{R}$. solani y Sclerotium rolfsii en pruebas in vitro. Lo anterior estaría dado entre otras características por su mayor o menor capacidad de producir enzimas específicas que degradarían la pared celular de $\boldsymbol{P}$. nicotianae (Pérez et al., 2002).

\section{Metabolitos volátiles}

Dentro del grupo de mutantes obtenidos mediante luz ultravioleta A y C, aparecen Th 11 A 20.1, Th 11 A 80.1, Th 11 A 160.1 y Th 12 A 10.1 con diferencias significativas con respecto a sus cepas parentales en el control de algunos de los patógenos en estudio (Tabla 3).

Aunque el porcentaje de inhibición fue menor en esta prueba, existen mutantes que reducen el crecimiento del patógeno, en porcentajes superiores al $40 \%$, es el caso de mutantes provenientes de $\boldsymbol{T}$. harzianum 11 controlando tanto, a la cepa de $\boldsymbol{R}$. solani 509, como a P. nicotianae (Tabla 3). KûcûK y Kivanc (2003), obtuvieron resultados similares, ya que el aislado de T. harzianum cepa T 19 controló bien a la cepa de Rhizoctonia solani, además, este mismo aislado inhibió de igual forma a patógenos como Fusarium oxysporum y $\boldsymbol{S}$. rolfsii mediante metabolitos volátiles.

\section{Metabolitos difusibles}

Se observó un aumento considerable en la inhibición de crecimiento miceliar por parte de los mutantes, 
Tabla 2. Antagonismo directo de mutantes de Trichoderma spp. sobre los patógenos Rhizoctonia solani GA 2-1, 4 y Phytophthora nicotianae

\begin{tabular}{|c|c|c|c|c|}
\hline \multicolumn{5}{|c|}{ Cepas de Patógenos y Porcentaje de Inhibición ${ }^{1}$} \\
\hline $\begin{array}{l}\text { Mutantes de } \\
\text { Trichoderma }\end{array}$ & $\begin{array}{r}\text { R. solani } \\
\text { (\%) }\end{array}$ & 509 & $\begin{array}{c}\text { R. solani } 618 \\
\text { (\%) }\end{array}$ & $\begin{array}{c}\text { P. nicotianae } \\
\text { (\%) }\end{array}$ \\
\hline T. harzianum $11^{*}$ & 55,35 & b & 81,38 a b & 68,4 a \\
\hline Th. 11 A 20.1 & 64,33 a & $\mathrm{b}$ & 72,58 & $37,15 \quad b$ \\
\hline Th. 11 A 80.1 & 73,35 a & & 85,55 a & $53,61 \mathrm{a} \mathrm{b}$ \\
\hline Th. 11 A 160.1 & 71,85 a & & 83,15 a & 53,17 a b \\
\hline T. harzianum 12* & 63,05 a & & 74,95 а & 77,2 а \\
\hline Th. 12 A 10.1 & 71,33 a & & 74,63 a & 63,52 a \\
\hline T. viride $32^{*}$ & 54,95 & b & 38,25 a & 0 a \\
\hline Tvi. NG 10 & 66,45 a & & 50,94 a & 0 a \\
\hline
\end{tabular}

11. Letras iguales en la columna significa que no existen diferencias significativas según la prueba de rango Múltiple de Tuckey, p ? 0,05. * Cepa silvestre (Padre)

Tabla 3. Metabolitos volátiles de mutantes de Trichoderma spp. sobre los patógenos $R$. solani GA 21, 4 y P. nicotianae

\begin{tabular}{|c|c|c|c|}
\hline \multicolumn{4}{|c|}{ Cepas de Patógenos y Porcentaje de Inhibición ${ }^{1}$} \\
\hline \multirow{2}{*}{$\begin{array}{c}\begin{array}{c}\text { Mutantes de } \\
\text { Trichoderma }\end{array} \\
\text { T. harzianum } 11^{*}\end{array}$} & $\begin{array}{c}\text { R. solani } 509 \\
(\%)\end{array}$ & $\begin{array}{c}\text { R. solani } 618 \\
(\%)\end{array}$ & $\begin{array}{c}\text { P. nicotianae } \\
(\%)\end{array}$ \\
\hline & 25,56 & 25,0 a & 8,33 \\
\hline & 41,11 a b & 20,0 a b & 47,22 a \\
\hline & 40,56 a b & 21,7 a b & 55,55 a \\
\hline Th. 11 & 42,78 a b & 16,7 a b c & 55,55 a \\
\hline T. harziant & 38,33 a & 32,8 a b & 19,4 a \\
\hline Th. 1 & 37,22 a b & 34,4 a & 47,2 a \\
\hline T. viri & 31,67 a & 5,00 a b & 0,00 a \\
\hline Tvi. $N$ & $11,67 \mathrm{~b}$ & 2,22 & $0,00 \quad a$ \\
\hline uckey & & * Сep & \\
\hline
\end{tabular}

ello se refleja en inhibiciones del $100 \%$ del crecimiento del patógeno. En el grupo de mutantes de nitroso guanidinio aparece Tvi NG 10 diferente estadísticamente a su cepa parental en el control de $\boldsymbol{R}$. solani 618 (GA 4) (Tabla 4). Para mutantes de luz ultravioleta se determinó que Th 11 A 20.1, Th 11 A 80.1 y Th 11 A 160.1, fueron estadísticamente diferentes a su cepa parental, siendo superiores en el control de los tres patógenos en estudio, mientras que Th 12 A 10.1 sólo presentó diferencias significativas con respecto a su cepa parental en el control de $\boldsymbol{R}$. solani 509 GA (2-1) y P. nicotianae (Tabla 4).

La mayoría de los mutantes seleccionados investigados lograron una inhibición superior al 80\%, incluso algunas alcanzaron el $100 \%$, siendo más efectivos que sus padres contra los patógenos en estudio. Lo anterior
Tabla 4. Metabolitos difusibles de mutantes de Trichoderma spp. sobre los patógenos Rhizoctonia solani GA 2-1, 4 y Phytophthora nicotianae

\begin{tabular}{|c|c|c|c|}
\hline \multicolumn{4}{|c|}{ Cepas de Patógenos y Porcentaje de Inhibición ${ }^{1}$} \\
\hline $\begin{array}{l}\text { Mutantes de } \\
\text { Trichoderma }\end{array}$ & $\begin{array}{c}\text { R. solani } 509 \\
\text { (\%) }\end{array}$ & $\begin{array}{c}\text { R. solani } 618 \\
\text { (\%) }\end{array}$ & $\begin{array}{c}\text { P. nicotianae } \\
\text { (\%) }\end{array}$ \\
\hline T. harzianum 11* & $70,6 \quad b$ & $89,4 \quad b$ & 95 \\
\hline Th. 11 A 20.1 & $100 \mathrm{a}$ & 99,4 a & 100 a \\
\hline Th. 11 A 80.1 & 100 a & $100 \mathrm{a}$ a & $100 \mathrm{a}$ \\
\hline T. h. 11 A 160.1 & 97,8 а & 98,3 а & $100 \mathrm{a}$ \\
\hline T. harzianum $12 *$ & $87,8 \quad b$ & $95 \mathrm{a} \mathrm{b}$ & 73,3 \\
\hline Th. 12 A 10.1 & $100 \mathrm{a}$ & $100 \mathrm{a}$ & 100 a \\
\hline T. viride $32 *$ & $20,6 \quad b$ & $0 \quad b$ & 19,4 a \\
\hline Tvi. NG10 & 6,11 & 10,6 a & 5,56 а \\
\hline
\end{tabular}

11. Letras iguales en la columna significa que no existen diferencias significativas según la prueba de rango Múltiple de Tuckey, p ? 0,05. * Cepa silvestre (Padre)

podría traducirse en un buen control en el campo de los patógenos evaluados.

En las tres pruebas de antagonismo efectuadas, llama la atención el bajo control obtenido por Tvi 32 y su mutante. Esto, se debería al efecto de la temperatura, ya que a $30{ }^{\circ} \mathrm{C} \mathbf{T}$. viride crece muy lentamente, debido a que su óptimo de crecimiento se encuentra a $22^{\circ} \mathrm{C}$ (Lieckfeldt et al., 1999; Lespinasse, 2003), mientras que los experimentos en esta investigación se realizaron a la temperatura óptima de los patógenos (García, 2001; Madrid, 2002).

\section{CONCLUSIONES}

De la investigación realizada y acorde con la metodología empleada, se desprenden las siguientes conclusiones:La mayoría de los mutantes de Trichoderma spp. investigados ejercieron un grado de control sobre el crecimiento de las cepas de $\boldsymbol{R}$. solani 509, 618 y P. nicotianae 699, demostrando una vez más las excelentes características biocontroladoras que habrían heredado de sus cepas parentales.

Los mejores mutantes según análisis de efectividadespectro de acción ( $\boldsymbol{R}$. solani 509, 618 y P. nicotianae 699), fueron Th 11 A 20.1, Th 11 A 80.1, Th 11 A 160.1 y Th 12 A 10.1 provenientes de las cepas de $\mathbf{T}$. harzianum 11 y 12 , respectivamente, mutadas con luz ultravioleta A, mientras que para mutantes de nitroso guanidinio el mejor mutante fue Tvi NG 10 proveniente de la cepa $\mathbf{T}$. viride.

Aunque los mutantes seleccionados resultaron poseer mayor actividad biocontroladora que las cepas silvestres (parentales), y además ser efectivos bioantagonistas contra los patógenos $R$. solani 509, 618 y P. nicotianae 699 en las pruebas de laboratorio, la 
expresión de las características biocontroladoras de estos mutantes dependen de una serie de factores que es necesario conocer y manejar. Por eso la necesidad de desarrollar nuevos y detallados estudios bajo condiciones de campo que aseguren tanto el éxito de las prácticas agrícolas como la sustentabilidad del sistema.

\section{REFERENCIAS}

Besoain, X.; García, R.; Raggi, C.; Oyanedel, J.; Montealegre, J.; Pérez, L. M. (2001). Biological control of Phytophthora parasitica in greenhouse tomatoes using Trichoderma harzianum Proceedings IOBC WPRS Bulletin vol. 24:103-107

Besoain, X.; Lefever, Ll.; Araya, A.; Montealegre, J.; Perez, L. (2004). Evaluación de mutantes de Trichoderma harzianum, producidos bajo la acción de luz negra y luz ultravioleta. XIV Congreso de la Sociedad Chilena de Fitopatología, Facultad de Ciencias Agrarias, Universidad de Talca. Chile, Nov.

CORFO. (1990). Enfermedades del tomate en invernadero frío. Valparaíso, Chile.

Dennis, C. \& Webster, J. (1971a). Antagonistic properties of speciesgroups of Trichoderma III. Hyphal interaction. Trans. Br. Mycol. Soc. 57:363-369

Dennis, C. \& Webster, J. (1971b). Antagonistic properties of speciesgroups of Trichoderma II. Production of volatile antibiotics. Trans. Br. Mycol. Soc. 57:41-48

Díaz, R. (1996). Comparación entre el uso de la solarización y el Bromuro de Metilo en el control de Rhizoctonia solani Kûhn y malezas en un suelo destinado al monocultivo del tomate. Memoria de Título Ing. Agr. Santiago, Universidad de Chile, Facultad de Ciencias Agrarias y Forestales.

Errázuriz, P. (2005). Impacto del Protocolo de Montreal: Los viudos del bromuro. Revista del campo 1514:4-5

Flint, M. L. \& Roberts, P. A. (1988). Using crop diversity to manage pest problems: some California examples. American Journal of Alternative Agriculture 3:164-167

García, S. R. (2001). Caracterización y uso in vitro de Trichoderma harzianum en la inhibición de Phytophthora nicotianae y su efecto en el control de pudrición al cuello en el cultivo de tomate
(Lycopersicon esculentum) bajo invernadero. Taller de Licenciatura, Universidad Católica de Valparaíso, Facultad de Agronomía.

INE. (2001). VI Censo agropecuario. Disponible en: http://www.ine.cl/ 34-censo/agropecuario.htm 8/06/2001. Citado el 10 de diciembre 2004

Kredics, L.; Antal, Z.; Manczinger, L.; Szekeres, A.; Kevei, F.; Nagy, E. (2003). Influence of environmental parameters on Trichoderma strains with biocontrol potential. Disponible en: http:// pubwww.srce.hr/ftbrfd/41-37.pdf. Citado el 22 de julio 2005

Kücük, C. \& Kivanc (2003). Isolation of Trichoderma spp. and determination of their antifungal, biochemical and physiological features. Disponible en: www.ansinet.org/fulltext/ajps/ajps34463471.pdf. Citado el 20 de junio 2005

Lespinasse, M. (2003). Caracterización de microorganismos antagonistas de patógenos vegetales, a través de su capacidad para excretar enzimas hidrolíticas de pared celular fúngica. Memoria de Título de Bioquímico. Santiago, Universidad de Chile Facultad de Ciencias Químicas y Farmacéuticas.

Lieckfeld, E.; Samuels, G.; Nirenberg, H. \& Petrini, O. (1999). Morphological and Molecular Perspective of Trichoderma viride: Is It One or Two Species?. Disponible en: http://aem.asm.org/cgi/content/ abstract/65/6/2418 Citado el 22 de julio 2005

Madrid, A. (2002). Control biológico de Rhizoctonia solani Kûhn en tomate (Lycopersicon esculentum) mediante el uso de Trichoderma spp. Memoria de Título Ing. Agr. Santiago, Universidad de Chile, Facultad de Ciencias Agrarias y Forestales.

Montealegre, J.; Pérez, M. L.; Herrera R.; Santander, C.; Velásquez, J.; Silva, P.; Besoain, X. (2002). Control of root rot fungi in tomatoes with Trichoderma harzianum, Bacillus lentimorbus and solarization under glasshouse and field conditions in Chile. Proceeding $7^{\circ}$ Meeting W. G. Biological Control of Fungal and Bacterial Plant Pathogens. Kusadasi, Turkey. May

Perez, L. M.; Besoaín, X.; Reyes, M.; Pardo, G.; Montealegre, J. (2002). The exppression of extracellular fungal cell wall hydrolytic enzymes in different Trichoderma harzianum isolates correlates with their ability to control Pyrenochaeta licopersici. Biological Research $35: 401-410$

Perez, L. M.; Besoaín, X.; Reyes, M.; Montealegre, J. (2004). Caracterización de mutantes de Trichoderma harzianum. (Resumen). XIV Congreso de la Sociedad Chilena de Fitopatología, Facultad de Ciencias Agrarias, Universidad De Talca. Chile, Nov.

Ristaino, J. \& Thomas, W. (1997). Agriculture, methyl bromide and the ozone hole can we fill the graps?. Plant Disease 81:964-977 